\title{
A SCALABLE APPROACH FOR SPATIO-TEMPORAL ASSESSMENT OF PHOTOVOLTAIC ELECTRICITY POTENTIALS FOR BUILDING FAÇADES OF ENTIRE CITIES
}

\author{
C. Braun ${ }^{1}$ \\ ${ }^{1}$ Luxembourg Institute of Science and Technology, L-4326 Esch/Alzette, Luxembourg - christian.braun@list.lu
}

Commission IV, WG IV/4ICWG

KEY WORDS: Renewable energy, Photovoltaic Potential, Building Façades, Smart City

\begin{abstract}
:
This paper describes results on the development of a completely FOSS-based approach assessing the electricity production potential by building façade PV. To estimate solar irradiation the hemispherical view-shed approach described by (Fu, 1999) was used. Combining it with an approach to dissect walls into regular 3D hyper-points (1-meter spacing) the sun visibility and the sky viewshed throughout the year are calculated. This results in global irradiation per hyper-point. To estimate the economic potential of each façade element an economic model was developed. This is driven by technical parameters of the installation, such as module efficiency, installation and maintenance costs, figures about payback tariffs and envisaged module lifetime. The overall result is a city-wide PV suitability and economic potential map of every building façade.

The processing is based on a city model in CityGML format using the 3DCityDB database and the spatial processing functionalities of PostGIS. A set of Python scripts has been developed as a central control instance and manage parallel processing of queries against the database to achieve scalability and improved performance. We run a case study with approximately 7000 single façade elements which are processed. Since we implemented a parallel computation of the façades running on an 80-core dedicated server machine, the completion for an entire city of about 3 million hyper-points points uses a decent amount of time for the given size of the data set. The chosen approach is highly scalable, robust and can be easily implemented through standard tools and libraries.
\end{abstract}

\section{INTRODUCTION}

The assessment of renewable energy potentials in urban environments gained a lot of interest in the recent decades due to $\mathrm{CO}_{2}$ reduction goals by cities, national policies as well as the energy efficiency directive by the EU. In combination with advances in data creation, handling and processing as well as the widespread definition of recent standards like the CityGML data model, new ways of modeling urban potentials have been developed. This lead to numerous approaches estimating rooftop solar photovoltaic (PV) production as described by (Freitas et al., 2015). However, in recent years due to research in building materials, the façades of buildings became more attractive and feasible for Photovoltaic electricity production as shown by (Fath et al., 2015) and (Catita et al., 2014).

First, a solar irradiation simulation study has been performed to know about the theoretical potential. Then a simplified economic potential assessment was carried out to map the citywide PV suitability and electricity production potential of every building façade. The area under study is the city of Esch-surAlzette in the Grand-Duchy of Luxembourg.

\section{STUDY AREA AND DATA}

Esch-sur-Alzette is located in the south-west of Luxembourg $\left(\mathrm{N} 49^{\circ} 30^{\prime}, \mathrm{O}^{\circ} 59^{\prime}\right)$ and is the second largest town in the country having a population of about 35000 inhabitants. It covers roughly $14 \mathrm{~km}^{2}$. The major area of the city is situated in a rather flat terrain with smaller hills stretching south-west to south-east of the city. The building density of the centre is quite high and the total number of buildings in the study area is about 7000 .
The study area was chosen because it is the place of work of the authors and with that comes the availability of additional important data sets from past projects of the institute. These are namely long-term and high resolution time series of irradiation data as well as a LiDAR data set. Furthermore, the Cadastre Agency of Luxembourg provides data about building footprints, contour lines and vertical height control points.

\section{SOLAR IRRADIATION CALCULATIONS}

It is well known that the global irradiation receiving the surface of the earth is the main driver for almost all biological and physical processes. Furthermore, on a global scale, the amount of received irradiation is depending on geographic latitude, seasons and day time as well as physical properties of the atmosphere like cloudiness or turbidity according to (Weischet, 1995).

Global irradiation is composed of three parts: a) direct irradiation, which depends on a direct unobstructed access to the sun; b) diffuse irradiation, which corresponds to the general ambient lighting conditions in shaded areas or under cloudy sky conditions and c) reflected irradiation, which originates from the reflections of the surrounding objects. Reflected irradiation is usually neglected because the amount is very low compared to the other two main components (see Fig. 1).

However, for assessment and the evaluation of potentials for PV in an urban context, topography plays a more distinctive role because it defines irradiation on the panel by slope and aspect of the panel itself as well as neighbouring objects might cast shadows and lower the amount of received irradiation. In an urban context cast shadows are even more important because of the often narrow areas between buildings and the then limited access 
to direct sunlight. The author made use of the concept of hemispherical view-shed models to assess irradiation on façades in this 'urban canyons'. First raster based models were already implemented in GIS software in the end of the 1990's by (Fu, Rich, 1999) but it is easily possible to use this approach and adapt it to vector features on a vertical façade or tilted roof surface and calculate the irradiation per point feature. First, the sky-view factor and the resulting diffuse irradiation per point feature is determined, then the direct irradiation is computed. The sum of direct and diffuse irradiation results in the global irradiation per year.

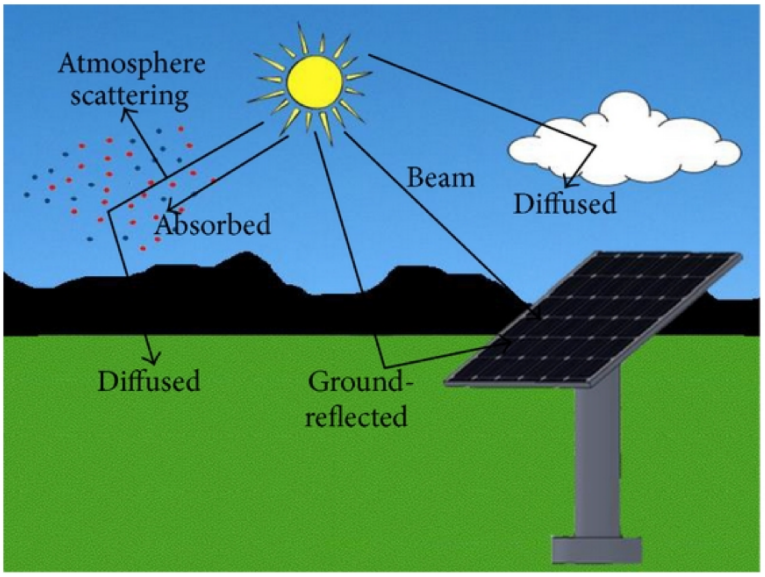

Figure 1. Components of global irradiation, source: (Lee et al., 2013)

\subsection{SKY VIEW FACTOR AND DIFFUSE IRRADI- ATION}

With the given view shed models it is necessary to first determine a sky view factor and then derive the diffuse irradiation for the point of observation. The sky view factor is a dimensionless parameter that defines the portion of the sky which is visible from a single point of observation. Its range is between 0 and 1 , where 1 would mean that the complete sky is visible from the point of observation. The computation is based on an artificially constructed hemisphere (see Fig. 2) with a regular spacing of azimuth and zenith angles for the centre points of the so-called sky sectors.

To finally assess diffuse irradiation a standard overcast diffuse model is used. Here the diffuse irradiation varies with the zenith angle of the flux originating from a weighted sky sector map. Each sector is weighted according to its position in the hemisphere (see Eq. 4).

For each sky sector the diffuse radiation at its centroid is calculated, integrated over the time interval and corrected by the gap fraction and angle of incidence using Eq. 1. Total diffuse irradiation $\left(D i f_{t o t}\right)$ for a ground location is the sum of the diffuse irradiation $\left(D i f_{\theta, \alpha}\right)$ from all sky sectors which are not obstructed from the point of view.

$\operatorname{Dif}_{\theta, \alpha}=R_{g l b} \cdot P_{d i f} \cdot$ Dur $\cdot S k y G a p_{\theta, \alpha} \cdot$ Weight $_{\theta, \alpha} \cdot \cos \left(\right.$ AngIn $_{\theta, \alpha}$

where:

$R_{g l b}=$ global normal radiation (see Eq. 2);
Figure 2. Constructed hemisphere showing centre-points of sky map sectors.

$P_{d i f}=$ proportion of global normal radiation flux that is diffuse; Dur = time interval for analysis, in case of the case study it is equal to 1 hour;

$S k y G a p_{\theta, \alpha}=$ gap fraction of the sky sector, in case of the case study it is equal to 1 ;

Weight $t_{\theta, \alpha}=$ proportion of diffuse radiation originating in a given sky sector relative to all sectors (see Eq. 4);

$A n g I n_{\theta, \alpha}=$ angle of incidence between intercepting surface and a given sky sector.

$$
R_{g l b}=\frac{S_{\text {const }} \sum\left(\tau^{m(\theta)}\right)}{1-P_{d i f}}
$$

where:

$S_{\text {Const }}=$ Solar flux outside the atmosphere at the mean earthsun distance, known as solar constant;

$\tau=$ transmissivity of the atmosphere;

$m(\theta)=$ relative optical path length: If $\alpha<80^{\circ}$ Eq. 3 has to be used. For $\alpha>80^{\circ}$ refraction has to be considered.

$$
m=\frac{E X P\left(-0.000118 \cdot E l e v-1.638 \cdot 10^{-9} \cdot \text { Elev }^{2}\right.}{\cos (\theta)}
$$

where:

$m(\theta)=$ relative optical path length;

$\theta=$ sky sector zenith angle;

Elev $=$ elevation above sea level in meters.

$$
\text { Weight }{ }_{\theta, \alpha}=\frac{2 \cos \theta_{2}+\cos 2 \theta_{2}-2 \cos \theta_{1}-\cos 2 \theta_{1}}{4 \cdot \operatorname{Div}_{a z i}}
$$

where:

$\theta_{1}$ and $\theta_{2}=$ bounding zenith angles of the sky sector;

Div $v_{a z i}=$ number of azimuthal divisions in the skymap.

\subsection{DIRECT IRRADIATION}

Total direct irradiation $\left(D i r_{t o t}\right)$ for a ground location is the sum of the direct irradiation $\left(\operatorname{Dir}_{\theta, \alpha}\right)$ from all sun map sectors (all 
sun positions throughout the year which are not obstructed by an obstacle). The direct irradiation from the sun map sector with a centroid at zenith angle $\theta$ and azimuth angle $\alpha$ is calculated using Eq. 5.

$\operatorname{Dir}_{\theta, \alpha}=S_{\text {Const }} \cdot \tau^{m(\theta)} \cdot \operatorname{SunDur}_{\theta, \alpha} \cdot \operatorname{SunGap}_{\theta, \alpha} \cdot \cos \left(\operatorname{AngIn}_{\theta, \alpha}\right)$

\section{where:}

$\operatorname{SunDur}_{\theta, \alpha}=$ time duration represented by the sun map sector, in case of the case study it is equal to 1 ;

$\operatorname{SunGap}_{\theta, \alpha}=$ gap fraction of the sun map sector, in case of the case study it is equal to 1 ;

AngIn $n_{\theta, \alpha}=$ angle of incidence between sun position and the axis to the surface normal.

$$
\begin{aligned}
& \text { AngIn } \operatorname{In}_{\theta, \alpha}=\arccos \left[\cos (\theta) \cdot \cos \left(G_{z}\right)+\sin (\theta) \cdot \sin \left(G_{z}\right) \cdot \cos \left(\alpha-G_{a}\right)\right] \\
& \text { where: } \\
& \text { AngIn } \operatorname{In}_{\theta, \alpha}=\text { angle of incidence between intercepting surface } \\
& \text { and a given sun position at zenith angle } \theta \text { and azimuth angle } \alpha ; \\
& G_{z}=\text { surface zenith angle; }
\end{aligned}
$$

\section{METHODS AND SOFTWARE}

The following subsections will describe the approach the author took to map the PV potential of building façades for the entire city area. The process involves the generation of CityGML ${ }^{1}$ (LoD 1) from LiDAR data and building footprints, the import

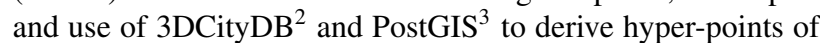
the building façades, the irradiation calculations and intersections with the terrain and surrounding buildings and finally the $\mathrm{PV}$ potential mapping per hyper-point.

\subsection{Generation of CityGML and import to 3DCityDB}

Since there is no detailed CityGML model (minimum LoD2) of the area of Esch-sur-Alzette the author required to create it himself. With the help of the 3Dfier ${ }^{4}$ software it is possible to generate valid CityGML data from building footprints (and other landscape elements) and a LiDAR point cloud. However, only LoD1 can be generated which is good enough for the use case of building façades. 3Dfier takes the $2 \mathrm{D}$ building polygons and lifts them to 3D according to their representation in the LiDAR point cloud. Different lifting options can be considered for CityGML thematic classes in the configuration file.

To get a more realistic view of the lighting conditions, the author decided to include a TIN (Triangular Irregular Network) relief of the surrounding landscape. The TIN relief is generated on the base of contour lines and vertical control points which are delivered by the Cadastre Agency. This is done with PostGIS functions to split the contour lines to smaller segments $(250 \mathrm{~m})$, derive its starting points and combine them with the level control points. With this collection of different punctual height information the TIN was generated by

\footnotetext{
${ }^{1}$ https ://www . opengeospatial .org/standards/citygml

${ }^{2}$ https://www.3dcitydb.org

${ }^{3}$ https://postgis.net

${ }^{4}$ https://github.com/tudelft3d/3dfier
}

ST_DelaunayTriangles. The resulting TIN and the 3D building polygons are shown in Fig. 3.

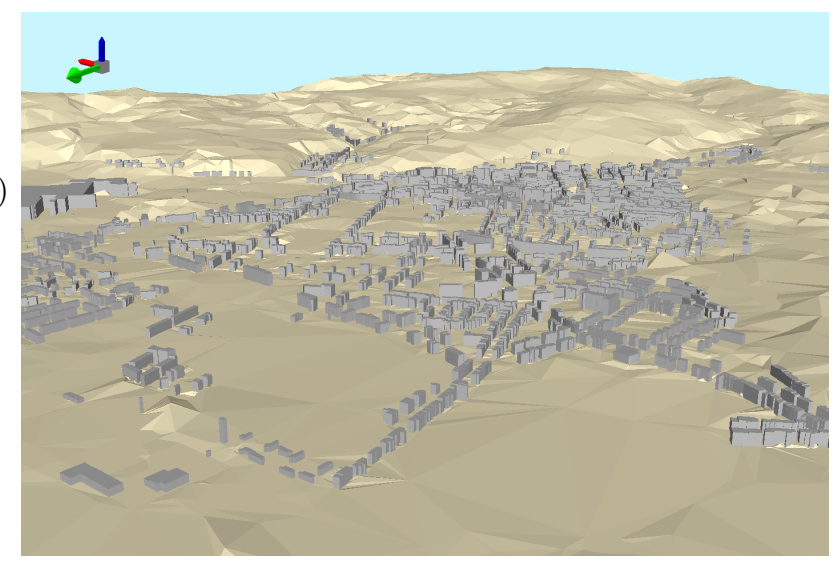

Figure 3. TIN and buildings ( $1.5 \mathrm{x}$ exaggeration, green arrow $=$ North)

\subsection{Dissection of Buildings and generation of 3D Hyper- Points}

According to a literature review it is often the case that the wall surfaces are dissected into a regular grid for processing. For each grid cell the centroid is used as a representation for the corresponding area of grid cell coverage and referred to as hyperpoints. In general, a point spacing of one meter is usually sufficient to represent the lighting conditions over the façade well enough if the computational time can be managed. The author followed the same approach and made use of the unified data model of 3DCityDB as well as the spatial processing capabilities of PostGIS. In a first step the segments of a building footprint have been extracted to generate single façade features, the azimuth of each façade was then calculated with ST_Azimuth and the height was extracted from the 3D wall representation. Since every terraced or end-of-terrace house is sharing a wall with the neighbouring house, the intersecting parts of the walls have to be identified in 2D and 3D space and then removed which leaves only segments of walls with their upper and lower height, which actually can be lit by the sun and represent a façade element. After this step, the remaining façade segments need to be dissected into 1 meter spaced points along their base and then followed by a vertically extruded copy to the respecting upper façade height. Finally, resulting in a regular spacing of 3D hyper-points (see Fig. 4). For this small to medium size city the processing of irradiation needs to be carried out for about 3 million hyper-points. Because all processing and derived hyper-points are based on the central geometry table of the data model, each hyper-point is still carrying the identifier of the building and its façade segment its belonging to, which is a prerequisite for the parallelisation of the irradiation calculations in the next steps.

\subsection{Irradiation calculations}

According to Section 3 the calculation of the global irradiation received by a hyper-point is two-fold and split in diffuse and direct irradiation processing. The sum of diffuse and direct are then resulting in the global irradiation per hyper-point for the entire year. To finish the calculations in a reasonable time frame it was necessary to find a way to parallelise the approach. This was accomplished by leveraging the processing functionalities of PostGIS and its support for spatial objects in combination with the 3DCityDB database and a set of Python scripts. 


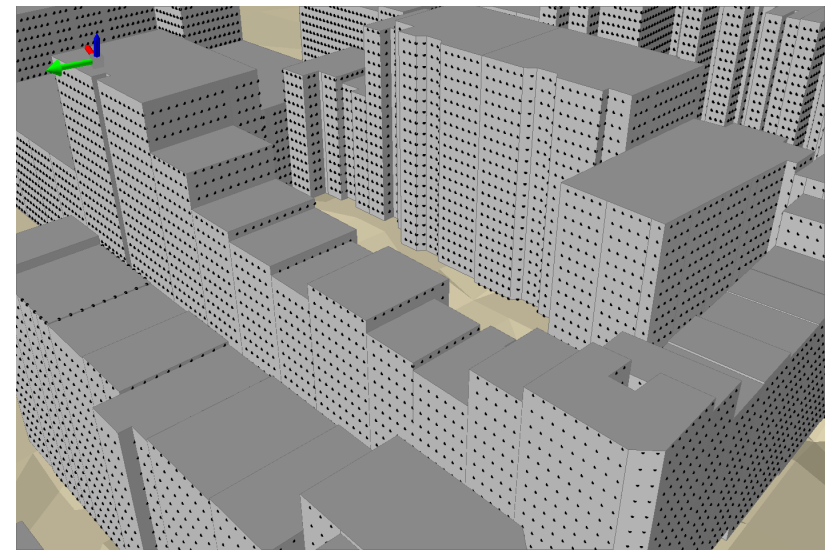

Figure 4. Hyper-Points, 1 meter spacing (1.5x exaggeration, green arrow $=$ North)

These are acting as a central "control instance". The scripts control the processing of ratios of direct and diffuse irradiation which the author could derive from own measurements as well as clear sky irradiation relying on the external "pvlib-pyton" Python library (Holmgren et al., 2018). Furthermore, Pythonbased scripts are used to manage parallel processing of queries against the database to achieve scalability and improved performance. The parallelisation is done by encapsulating each façade element and its corresponding hyper-points and send the necessary SQL code to the database in a dedicated connection. A more detailed implementation of the calculations is given in the next two Sections.

4.3.1 Diffuse Irradiation First, an artificial hemisphere was created with azimuth angles in $15^{\circ}$ steps and zenith angles in $9^{\circ}$ steps accordingly for the centre points of the sky sectors originating in the centre of Esch-sur-Alzette. For the radius $100 \mathrm{~km}$ was used (see Fig. 2). This originates in $240 \mathrm{sky}$ sectors which are weighted according to their position on the hemisphere. For each imaginary line between a hyper-point and a sky sector intersections are tested in the following calculations. Whereas sky sectors that are located on the back side of a wall element are ignored. This is done by checking where the incidence angle to the wall orientation is smaller than $90^{\circ}$. Afterward, the first test for intersection is done by ST_3D_Intersects with the surrounding TIN representing the terrain. If this test is negative than a second intersection test is processed to see whether the hyper-point is sunlit or obstructed by a neighbouring building. This leads to the sky view factor which determines overall diffuse irradiation.

In a next step $R_{g l b}$ and $P_{d i f}$ (see Eq. 1) were needed to be estimated on an hourly time step throughout the year. $R_{g l b}$ was generated by pvlib-python. $P_{d i f}$ is generated from a long term irradiation measurement station which is run by the author's institute in the centre of Esch-sur-Alzette. With information about weighting and the angle of incidence of the sky sectors the diffuse irradiation was calculated only for the hyper-points which have direct view access to the hemisphere, thus not being obstructed by terrain or other obstacles. The data about diffuse irradiation is then aggregated by hyper-point for the entire year and stored in the resulting table.

4.3.2 Direct Irradiation While there are 240 sky sectors to test for intersection it is necessary to calculate intersection tests for all the possible sun positions. If the desired resolution is a one hour time step this is leading for the entire year to about theoretical 4500 intersection tests (only counting from sunrise to sunset). To further reduce the number of intersection tests a test for façade orientation is performed which reduces the number of tests significantly already. A further reduction is achieved by the number of days the sun positions are calculated. The difference in the sun position is greater from hour to hour during the day than for the same hour on consecutive days. According to (Zahn, 2015) the author has chosen an interval of 10 days which should still give reasonable results and further reduces overall computation time.

As with the calculation of the diffuse irradiation, a imaginary line between a hyper-point and a sun position is drawn and tested for intersection with the terrain and neighbouring buildings. According to Eq. 5 the direct irradiation is computed and summed up for all non-obstructed combinations of a hyperpoint to sun position.

This workflow is as well parallelised in the same way as for the diffuse irradiation.

Finally, the global irradiation per hyper-point is the sum of diffuse and direct irradiation.

\subsection{Economic Potential calculations}

With information about global irradiation per hyper-point and general assumptions about panel parameters and economic parameters, a simple economic potential analysis can be carried out according to $\mathrm{Eq} 8$. The result would be the minimum required global irradiation per year to cover the costs of an installation over the given envisaged lifetime which is a relevant figure for a potential investor or building owner.

$$
\begin{aligned}
\text { MinIrra }_{\text {global }} & =\frac{\text { Electricity }_{\text {costcover }}}{E f f_{\text {overall }}} * 100 \\
\text { Electricity }_{\text {costcover }} & =\frac{\text { Cost }_{\text {overall }}}{\text { Paybackprice } \cdot \text { Lifetime }_{\text {econ }}}
\end{aligned}
$$

Cost $_{\text {overall }}=\frac{\text { Cost }_{\text {panel }}}{\text { Area }_{k W p}}+\frac{\text { Cost }_{\text {panel }}}{\text { Area }_{k W p}} \cdot \frac{\text { Cost }_{\text {annual }} \cdot \text { Lifetime }_{\text {econ }}}{100}$

$$
\text { Area }_{k W p}=1 /\left(E f f_{\text {overall }} / 100\right)
$$

$$
E f f_{\text {overall }}=E f f_{\text {panel }} \cdot\left(100-\text { Loss }_{\text {sys }}\right) / 100
$$

where:

Loss $_{\text {sys }}=$ Loss of entire system installation;

Ef $f_{\text {panel }}=$ panel efficiency;

Cost $_{\text {panel }}=$ cost of PV panels per $m^{2}$;

Cost $_{\text {annual }}=$ annual operation costs in percent of installation costs;

Lifetime $_{\text {econ }}=$ envisaged lifetime of the installation;

Paybackprice $=$ Payback-price of energy fed back into the grid in Euro.

Finally, the hyper-points below the minimum irradiation margin are removed from the aggregation of the façade surface they are belonging to and an average cost in Euro/kWh is assigned to the façade element. This is resulting in a qualitative and quantitative mapping of façades for the entire city. 


\section{RESULTS}

\subsection{Irradiation calculations}

The generated results for the components of irradiation and hence global radiation are satisfactory and in range with to be expected mean irradiation values for this region and climatic conditions. Peak values are reached with up to $1100 \mathrm{kWh} / \mathrm{a}$. Hyper-points in higher south-facing façade areas with a lot of sunlit hours per year gain usually more than $700 \mathrm{kWh} / \mathrm{a}$ (see Fig. 5).

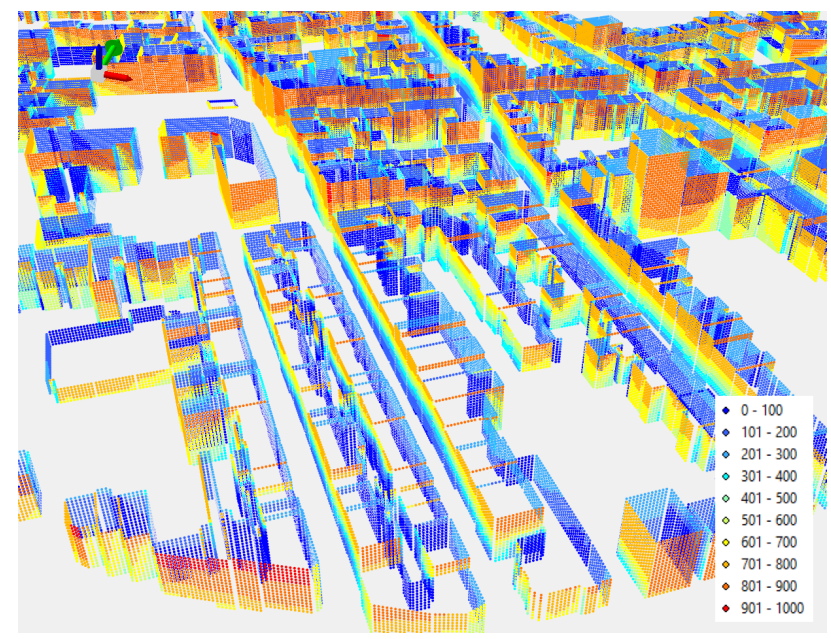

Figure 5. Global irradiation per hyper-point [kWh/a] (clipping, south-west city area)

The lowest values are ranging from 100-200 kWh/a for the diffuse components (see Fig. 6) in lower wall areas. The homogeneous pattern is because since diffuse irradiation is more or less only determined by the sky view factor. Thus lower parts of façades with limited visibility are getting lower values.

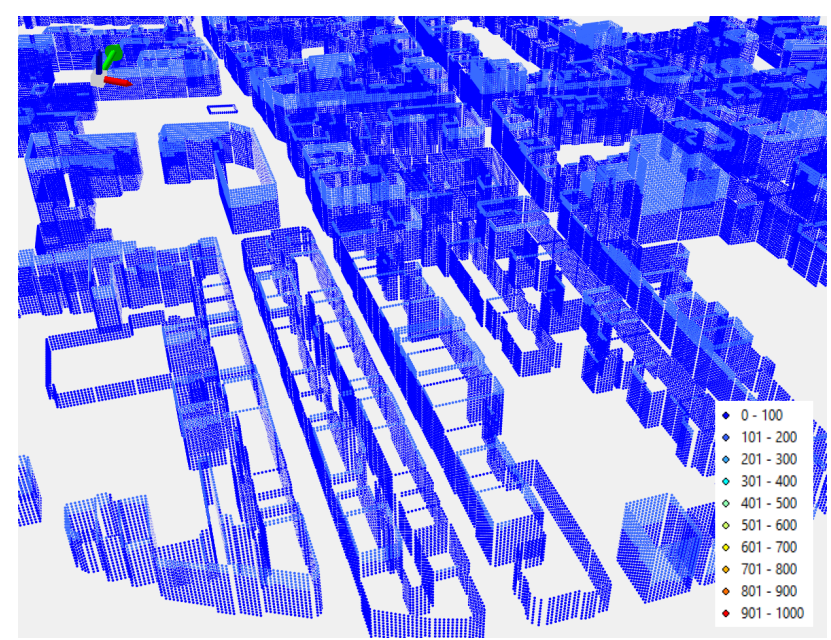

Figure 6. Diffuse irradiation per hyper-point [kWh/a]

Direct irradiation is up to $700-800 \mathrm{kWh} / \mathrm{a}$ in higher and southwards exposed wall areas (see Fig. 7). It is also clearly visible that the intersection tests with buildings and terrain are working due to the patterns of different lighting conditions in 'urban canyons' as well as respecting sun positions because of low values on the northern facing side of buildings.

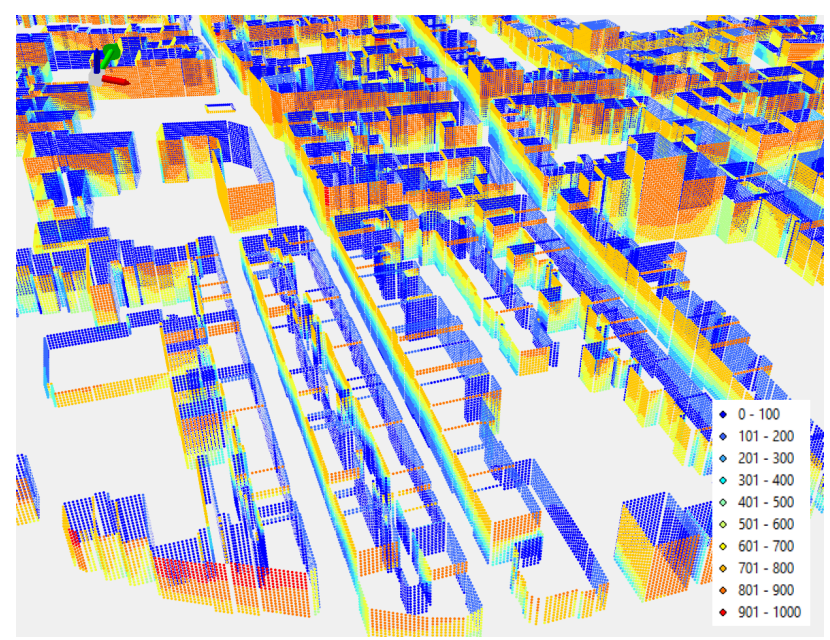

Figure 7. Direct irradiation per hyper-point [kWh/a]

\subsection{Economic Potential calculations}

The scenario of an examplary economic potential calculation was carried out with arbitry values for panel technologies and economic parameters for Eq. 7 - 11 where:

Loss $_{\text {sys }}=15$ percent;

$E f f_{\text {panel }}=15$ percent;

Cost $_{\text {panel }}=2000 \mathrm{Euro} / \mathrm{m}^{2}$;

Cost $_{\text {annual }}=1.5$ percent of installation cost;

Lifetime $_{\text {econ }}=20$ years;

Paybackprice $=0.245$ Euro.

This leads to results shown in Fig. 8 and 9. Dark green façades would be paid back in the envisaged 20 years or earlier of system lifetime, while light green would take a bit longer. Orange and red coloured façades would not be considered in a first approach because of their high electricity production costs for the given set of parameters thus being not economically exploitable.

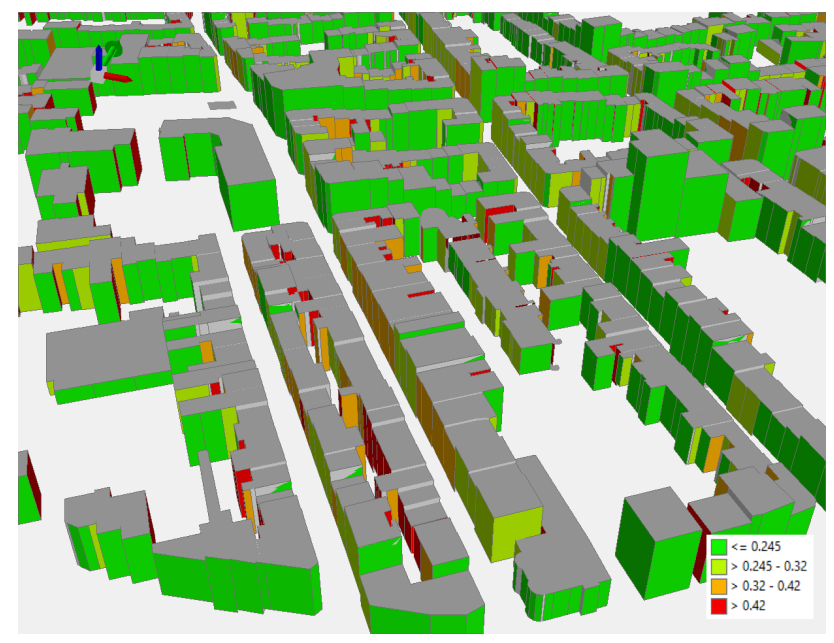

Figure 8. Cost of generated electricity per façade in [Euro/kWh] (clipping, south-west city area)

\section{CONCLUSION}

One of the major bottlenecks of the approach is testing for intersection with surrounding buildings for sun visibility for every 


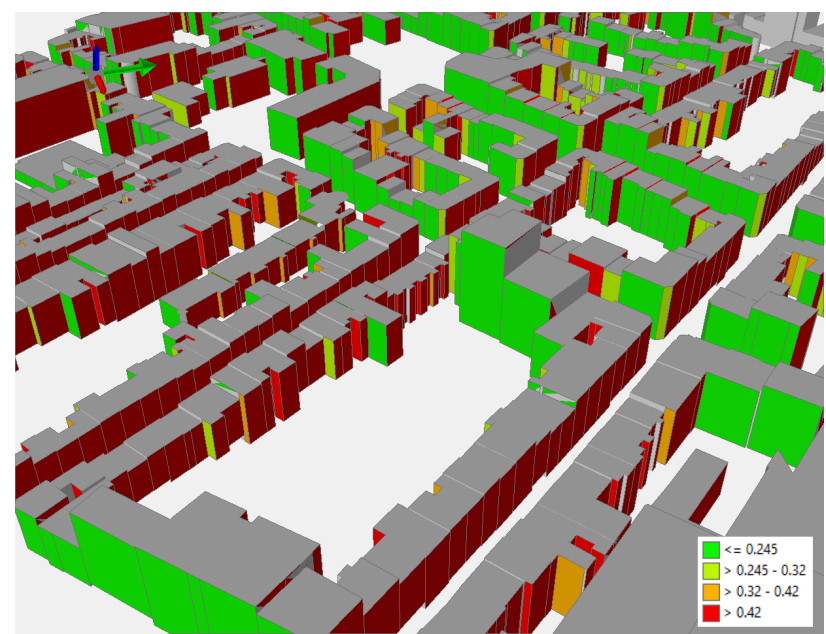

Figure 9. Cost of generated electricity per façade in [Euro/kWh] for a different point of view (clipping, south-west city area)

hyper-point per timestamp. This takes per façade element several minutes to process depending on the number of hyperpoints per wall. Since parallel processing of the façade elements is implemented and running on an 80-core dedicated server machine, the completion for an entire city of 3 million generated hyper-points uses a decent amount of time (about 15 days) for the given size of the data set and complexity of processing.

The described approach is highly scalable through parallelisation and it can be easily implemented through standard tools and libraries. This opens up possibilities for distributed approaches using multiple database servers for even better scalability.

However, all this is not taking parts of façades into account where no installation would possible like window or door openings. This could be addressed as well in further developments where specific parts of the buildings are not taken into account or with higher LoD city models.

\section{ACKNOWLEDGEMENTS}

This research was carried out and financed in the framework of the 'SECuRe - An interoperable geospatial Smart Energy Cities and Regions Platform for Luxembourg' project. SECuRe is a collaboration between the Fondation Enovos and Luxembourg Institue of Science and Technology.

\section{REFERENCES}

Catita, C., Redweik, P., Pereira, J., Brito, M., 2014. Extending solar potential analysis in buildings to vertical façades. Computers \& Geosciences, 66, 1 - 12.

Fath, K., Stengel, J., Sprenger, W., Wilson, H., Schultmann, F., Kuhn, T., 2015. A method for predicting the economic potential of (building-integrated) photovoltaics in urban areas based on hourly Radiance simulations. Solar Energy, 116, 357 - 370.

Freitas, S., Catita, C., Redweik, P., Brito, M., 2015. Modelling solar potential in the urban environment: State-of-the-art review. Renewable and Sustainable Energy Reviews, 41, 915 931.
Fu, P., Rich, P., 1999. Design and implementation of the Solar Analyst: an ArcView extension for modeling solar radiation at landscape scales. Proceedings of the 19th Annual ESRI User Conference.

Holmgren, W., Hansen, C., Mikofski, M., 2018. Pvlib Python: a Python Package for Modeling Solar Energy Systems. J. Open Source Software, 3, 884.

Lee, J., Abd R., N., Al-Turki, Y., 2013. Performance of DualAxis Solar Tracker versus Static Solar System by Segmented Clearness Index in Malaysia. International Journal of Photoenergy, 2013, 1-13.

Weischet, W., 1995. Einführung in die Allgemeine Klimatologie: Physikalische und meteorologische Grundlagen. Teubner Verlag, Stuttgart.

Zahn, W., 2015. Sonneneinstrahlungsanalyse und informationsanreicherung von großen 3d-stadtmodellen im citygmlschema. Masterarbeit, Technische Universität München. 